\title{
Incentives in Decentralized Random Matching Markets
}

\author{
Joana Pais* \\ UECE - Research Unit on Complexity in Economics \\ ISEG/UTL
}

October 2005

\begin{abstract}
${ }^{*}$ I have received useful comments from Coralio Ballester, Antoni Calvó, Gabrielle Demange, Lars Ehlers, Guillaume Haeringer, Bettina Klaus, Flip Klijn, Antonio Romero-Medina, and Alvin Roth. I am especially grateful to Jordi Massó. I acknowledge the financial support from the Fundação para a Ciência e a Tecnologia through grant $\mathrm{n}^{o} \mathrm{SFRH} / \mathrm{BD} / 5308 / 2001$ and from the Spanish Ministry of Science and Tecnology through grant BEC 2002-002130. Address: Instituto Superior de Economia e Gestão, Universidade Técnica de Lisboa, R. Miguel Lupi, 20, 1249-078 Lisboa, Portugal. E-mail: jpais@iseg.utl.pt
\end{abstract}




\begin{abstract}
Decentralized markets are modeled by means of a sequential game where, starting from any matching situation, firms are randomly given the opportunity to make job offers. In this random context, we prove the existence of ordinal subgame perfect equilibria where firms act according to a list of preferences. Moreover, every such equilibrium preserves stability for a particular profile of preferences. In particular, when firms act truthfully, every outcome is stable for the true preferences. Conversely, when the initial matching is the empty matching, every stable matching can be reached as the outcome of an ordinal equilibrium play of the game.
\end{abstract}

JEL Classification: C78; J44

Keywords: Matching Markets; Stability; Random Mechanisms 


\section{Introduction}

The study of centralized markets has been privileged in the two-sided matching literature. The introduction of centralized matching procedures in markets that experienced certain kinds of failures is partially responsible for such dedication. In fact, a number of markets - for physicians, lawyers, dentists, and osteopaths, among others-have adopted central clearinghouses after periods of uncontrolled unraveling of appointment dates and chaotic recontracting. ${ }^{1}$ These markets now work by having each agent of the two sides of the market submit a rank ordered preference list of acceptable matches to the central clearinghouse, which then produces a matching by processing all the preference lists according to an algorithm. Roth (1984a, 1991) showed that the algorithms used in most of the successful clearinghouses roughly follow the lines of Gale and Shapley's deferred acceptance algorithm (Gale and Shapley, 1962). This procedure generates a matching of workers to positions that is stable in terms of the submitted preferences in the sense that no worker and firm that are not matched to each other would prefer to be so matched. ${ }^{2}$

In contrast, decentralized markets have received relatively little attention. ${ }^{3}$ The exact set of rules that governs a centralized market, making it particularly amenable to analysis, is no longer present when matching is organized in a decentralized way. Moreover, decentralized markets involve different strategic issues from those of centralized markets. In fact, when a clearinghouse exists, agents must simply decide what preference lists to submit to the matchmaker, after which the match is created. However, in a decentralized market agents do not submit lists; instead,

\footnotetext{
${ }^{1}$ See Roth and Xing (1994) and Niederle and Roth (2003).

${ }^{2}$ See Roth and Sotomayor (1990) for a comprehensive study of two-sided matching markets.

${ }^{3}$ There are notable exceptions, namely Blum, Roth, and Rothblum (1997), Haeringer and Wooders (2004), Roth and Vande Vate (1991), Roth and Xing (1997), among others.
} 
they can decide, after each interview or telephone call what to do next. The size of the strategy space is thus extremely large and has precluded analysis by means of standard matching tools.

The purpose of this paper is to apply the extremely simple marriage model to the study of decentralized labor markets. In this model, agents' preferences are ordinal in nature. Hence, we merely take for granted their ability to order the potential working partners, without assuming that they are capable of giving cardinal content to their preferences. ${ }^{4}$ The starting point of the analysis is any matching situation, providing a framework to the study of both entrylevel and senior level markets. The matching process is then modeled as an extensive form game, where firms sequentially offer their positions. Clearly, decentralized decision making in complex environments may introduce randomness in the order in which offers are made. The speed of the mail, the telephone network, or the internal structure of firms making some react faster than others determine the success in establishing communication with the desired workers. Such inherently uncertain features of the market are modeled here as chance moves that determine the order of play. Hence, at each moment in time, any firm- even if already matched - is randomly selected and given the opportunity to offer its position to a worker. This worker compares it with any offer he may be holding and rejects one, while (temporarily) holding the other, pending the possible arrival of even better offers. Note that only firms have the initiative to make proposals or to cease a working relationship, while workers undertake the more passive role of reacting to offers. We assume that, once rejected, the firm is not willing to propose to the same worker again, but it may obviously offer its position to a different worker

\footnotetext{
${ }^{4}$ It follows that monetary transfers are embodied in agents' preferences, i.e., there is more to a job than just a salary.
} 
when given the opportunity to act. ${ }^{5}$

In a decentralized labor market, informal rules and practices govern the process of making proposals, and also their comparison, acceptance, or rejection. There are thus different options when it comes to modeling such elements of market culture which, together with the assumptions taken on what agents know when taking decisions, play an important role in influencing the results. Two closely related papers are worth mentioning. Haeringer and Wooders (2004) model decentralized markets in which only firms make job offers, agents' decisions may be irreversible - once a proposal is accepted, the agents involved simply leave the market - and agents hold a lot of information on the history of the game. ${ }^{6}$ There is no uncertainty whatsoever, suggesting that markets are small worlds. In contrast, Blum, Roth, and Rothblum (1997) model a senior level labor market where agents are poorly informed - each firm knows which of her offers have been rejected and by whom, and each worker recalls all the proposals he obtained - and there is no commitment. Nevertheless, only firms with vacant positions are allowed to make proposals: firing a worker is simply too costly. In this paper, no such restriction is imposed, i.e., any firm, even if matched, can hire a new worker or simply fire its current match. There is no commitment: agents are greedy and always willing to look for a better partner. Moreover, agents may hold different degrees of information on the actions of the others, ranging from the imperfect information scenario in Blum, Roth, and Rothblum (1997) to knowing every proposal made, accepted, and rejected. Finally, we extend the analysis to the case in which every agent, firm or worker, may have the initiative to either break the current

\footnotetext{
${ }^{5}$ It does not appear that allowing for any finite number of repeated proposals would materially change the validity of the results that follow.

${ }^{6}$ Throughout the paper, information is assumed to be perfect or almost perfect: agents on each side of the market may ignore some actions of their peers.
} 
match or start a new working relationship. Hence, this paper does not compete with the cited literature, but rather complements it by providing yet another perspective on the functioning of decentralized markets.

In our setting, the random order in which firms are selected introduces some uncertainty in which matchings are achieved. In fact, it may happen that starting with the same initial matching, different plays of the game yield different outcomes for the same strategy profile. Since preferences are ordinal, in order to compare different probability distributions over matchings, we use a solution concept based on first-order stochastic dominance. The notion of ordinal Nash equilibrium guarantees that each agent is an expected utility maximizer for every utility representation of his preferences. ${ }^{7}$ We go beyond this concept to account for the dynamic nature of the game and characterize subgame perfect ordinal Nash equilibria. Despite the strength of this concept, we prove the existence of subgame perfect ordinal Nash equilibria and, in particular, equilibria where firms use preference strategies (i.e., strategies that can, up to some point, be identified with a list of preferences). On the other hand, every such equilibrium delivers matchings that are stable with respect to a particular profile of preferences. This has two appealing implications. First, for any equilibrium where firms adhere to preference lists, all outcomes are such that the set of unmatched agents is the same. Second, in the particular case that firms act according to their true preferences, stability with respect to the true preferences is guaranteed in a subgame perfect ordinal Nash equilibrium. This provides an explanation for the success of some decentralized labor markets. In fact, if we expect equilibria where firms act straightforwardly to prevail, only stable matchings are obtained and no individual agent

\footnotetext{
${ }^{7}$ This concept was introduced in d'Aspremont and Peleg (1988); it has been used in the context of voting theory in Majumdar and Sen (2004) and in matching markets in Ehlers and Massó (2003), Majumdar (2003), and Pais (2004).
} 
or pair of agents (consisting of a firm and a worker) will have the incentive to circumvent the matching. Moreover, using a list of preferences as a strategy or revealing the true preferences can be justified in some settings. The decisions of a firm do not usually reflect the opinion of a single individual; instead, such actions embody a complex process of assembling the opinions of several individuals. We may conjecture that establishing a list of candidates and using it as guidance is - despite the apparent myopia - a more plausible form of behavior than deciding, at each moment in time, whom to propose to. In addition, in some settings firms obey objective criteria to admit workers, so that strategic behavior on the firms' side looses its meaning. The (partially) converse statement holds when we start from a situation where all agents are unmatched: every stable matching for the true preferences can be reached as the outcome of an equilibrium play where firms act straightforwardly according to their true preferences.

The paper is organized as follows. In Section 2 we introduce the matching model, and review some results on matching markets. We formally present the model in Section 3. In Section 4 we turn our attention to questions related to individual decision making and characterize equilibria. Some results and underlying assumptions are discussed in Section 5. We conclude in Section 6. Some proofs can be found in the Appendix.

\section{The Marriage Model}

Consider two finite and disjoint sets $F=\left\{f_{1}, \ldots, f_{n}\right\}$ and $W=\left\{w_{1}, \ldots, w_{p}\right\}$, where $F$ is the set of firms and $W$ is the set of workers. We let $V=W \cup F$ and sometimes refer to a generic agent by $v$, while $w$ and $f$ represent a generic worker and firm, respectively. Each agent has a strict, complete, and transitive preference relation over the agents on the other side of the market and the perspective of being unmatched. The preferences of a firm $f$, for example, can 
be represented by $P_{f}=w_{3}, w_{1}, f, w_{2}, \ldots, w_{4}$, indicating that $f$ 's first choice is to be matched to $w_{3}$, its second choice is $w_{1}$ and it prefers remaining unmatched to being assigned to any other worker. Sometimes it is sufficient to describe only $f$ 's ranking of workers it prefers to remaining unmatched, so that the above preferences can be abbreviated as $P_{f}=w_{3}, w_{1}$. Let $P=\left(P_{f_{1}}, \ldots, P_{f_{n}}, P_{w_{1}}, \ldots, P_{w_{p}}\right)$ denote the profile of all agents' preferences; we sometimes write it as $P=\left(P_{v}, P_{-v}\right)$ where $P_{-v}$ is the set of preferences of all agents other than $v$. Further, we may use $P_{U}$, where $U \subseteq V$, to denote the profile of preferences $\left(P_{v}\right)_{v \in U}$. We write $v^{\prime} P_{v} v^{\prime \prime}$ when $v^{\prime}$ is preferred to $v^{\prime \prime}$ under preferences $P_{v}$ and we say that $v$ prefers $v^{\prime}$ to $v^{\prime \prime}$. We write $v^{\prime} R_{v} v^{\prime \prime}$, when $v$ likes $v^{\prime}$ at least as well as $v^{\prime \prime}$ (it may be the case that $v^{\prime}$ and $v^{\prime \prime}$ are the same agent). A worker is acceptable if the firm prefers to employ him rather than having its position unfilled; similarly, a firm is acceptable to a worker if he prefers occupying its position, rather than being unemployed.

Formally, a marriage market is a triple $(F, W, P)$. An outcome for a marriage market, a matching, is a function $\mu: V \longrightarrow V$ satisfying the following: (i) for each $f$ in $F$ and for each $w$ in $W, \mu(f)=w$ if and only if $\mu(w)=f$; (ii) if $\mu(f) \neq f$ then $\mu(f) \in W$; (iii) if $\mu(w) \neq w$ then $\mu(w) \in F$. If $\mu(v)=v$, then $v$ is unmatched under $\mu$, while if $\mu(w)=f$, we say that $f$ and $w$ are matched to one another. A description of a matching is given by $\mu=\left\{\left(f_{1}, w_{2}\right),\left(f_{2}, w_{3}\right)\right\}$, indicating that $f_{1}$ is matched to $w_{2}, f_{2}$ is matched to $w_{3}$ and the remaining agents in the market are unmatched. A matching $\mu$ is individually rational if each agent is acceptable to its partner, i.e., $\mu(v) R_{v} v$, for all $v \in V$. We denote the set of all individually rational matchings by $\operatorname{IR}(P)$. Two agents $f$ and $w$ form a blocking pair for $\mu$ if they prefer each other to the agents they are actually assigned to under $\mu$, i.e., $f P_{w} \mu(w)$ and $w P_{f} \mu(f)$. A matching $\mu$ is stable if it is individually rational and it is not blocked by any pair of agents. We denote the 
set of all stable matchings by $S(P)$.

\section{The Decentralized Job Matching Game}

\subsection{Description of the Game}

In this section, we define the Decentralized Game. The game is given by a market $(F, W, P)$ and an initial matching $\mu^{I}$. In general, we consider $\mu^{I}$ to be individually rational under the true preferences. The rules of the game are as follows.

The game begins with a node at which nature chooses a sequence of firms at random. Each sequence corresponds to an order at which firms are given the opportunity to make proposals. Following nature's move, the first firm in the selected sequence has the chance to make a proposal. If unmatched under $\mu^{I}$, the firm may propose to any worker or pass its turn. If matched under $\mu^{I}$, it may simply fire its initial partner, propose to a different worker, or pass its turn and keep the initial partner.

In the case that a proposal is actually made, the game continues by having the proposed worker deciding whether to accept or to reject the offer. If he accepts, a new matching is formed where this worker and the proposing firm are together and their previous partners, if

any, are unmatched. If he rejects, $\mu^{I}$ goes on unchanged. In the case that the firm simply chose to fire its initial worker, a new matching is formed where the firm and its former partner are unmatched, whereas if the firm chose "pass," the initial matching is preserved.

The second firm then moves and the game continues by giving firms the opportunity to make offers, in accordance with the order of the sequence. Each time a firm is called to play, the available moves depend on whether its position is vacant or not. If vacant, the firm may 
propose to any worker to whom it has not proposed before or simply pass its turn. Otherwise, it may fire the worker it holds, propose to a worker different from its current match and from any worker it has already proposed to, or pass its turn. When a worker receives a proposal, he may accept the offer or reject it and keep his former partner.

The game continues as long as there is at least one firm wishing to make a new offer or to fire the incumbent worker. As soon as every firm in the market sequentially passes its turn, the game ends.

Two remarks are in order. First, the fact that firms are reluctant to repeating offers guarantees that every play of the game ends in a finite number of steps. Still, relaxing this simplifying assumption and allowing for a finite number of repeated offers would not compromise the results. Second, a matched worker is only allowed to reject his current position if he obtains and accepts an alternative offer. This is a one-side-proposing game: workers do not have the spirit to make offers or even to quit their current job. In Section 5, we discuss the consequences of having both firms and workers playing an active role.

To complete the description of the game, we still have to specify the information that each agent possesses throughout the game. It is sensible to assume that in labor markets where myriads of firms and workers interact, each agent only becomes aware of events as they directly impinge on him. In the particular case of a firm, this means that it learns only if the proposal it made was accepted or rejected, or if its position became vacant. Hence, a firm's information set is defined by its initial partner and an ordered list of workers to whom it proposed, along with their reactions. Similarly, a worker is only aware of events that directly affect him. A worker's information set is identified by his initial position, as well as an ordered list of proposals received, his own responses, and firings. The initial chance move is never 
observable. $^{8}$

\subsection{Chance Move}

Let us now focus on nature's move. At each moment in time, a randomly selected firm is given the chance to play. This random selection should not be interpreted merely as every firm having equal probability of proposing at each step. It may reflect some institutionaland perhaps inherently uncertain - features of the market which are not modeled. In fact, in decentralized markets matching is performed over the telephone network, using the mail, or through the Internet. In such environments, randomness determines the order in which agents communicate: it may depend on which telephone call goes through, on the speed of the mail, or on how fast firms react to eventual proposals. Or it may even be the case that there exists a natural order in which firms are expected to propose - firms that have potentially more to gain will certainly devote more resources into finding the right worker for their position and are, therefore, more likely to make offers.

To be precise, the game starts with a lottery prescribing a sequence of firms that defines the subsequent moves. A sequence corresponds to one of the innumerable possible orders in which firms are allowed to act. We assume that every sequence is infinite and that, in each sequence, every single firm appears infinitely many times. We also assume that every sequence has positive probability of occurring. The sample space over which this probability distribution is defined is denoted by $O$ and $o$ is an arbitrary sample point, a sequence of firms.

\footnotetext{
${ }^{8}$ Such low information environment may be enriched. It may be the case that agents learn of the actions of the others, even though they are not immediatly affected by them. The validity of the results that follow will be discussed for broader information structures.
} 
Note that, even though we consider infinite sequences of firms, every play of the game ends in finite time. In fact, as firms are only allowed to propose to each worker once and, obviously, firing is possible only if matched, the moment comes when every firm chooses to pass its turn if called to play, either because keeping the incumbent worker is part of its strategy, or because passing is the only available action. Moreover, the fact that a firm appears infinitely many times in each sequence guarantees that this moment comes and the firm is actually called to play. The end is then reached for every play of the game.

\subsection{The Strategy Space}

In what follows, we will describe agents' strategies and introduce some notation. A player's strategy in the Decentralized Game complies with the usual definition of behavioral strategy in an extensive form game, i.e., a plan of action for each information set where he is called to act. However, in the context of a matching market there is a class of strategies worth emphasizing, strategies that resemble those used in a centralized market. Following Blum, Roth, and Rothblum (1997) we will call these strategies "preference strategies." Such strategies obey a consistency criterion in which agents decide how to move at any information set basing on a list of preferences, including those information sets that would not be reached had that list actually been used. Hence, deviations are regarded as temporary mistakes and further moves fit in the original list. To make things clear, when using a preference strategy, a firm selects an ordered list of potential matches and, whenever called to propose, makes the offer to the best worker on its list to whom it has not proposed before; likewise, a worker decides whether to accept or to reject a new proposal by comparing it with his current position on his list.

Even though the lists of preferences that serve as guidance do not have to faithfully reveal 
agents' true preferences, the set of preference strategies represents merely a small part of the set of feasible strategies. ${ }^{9}$ For example, a worker $w^{\prime}$ s strategy of accepting only the first proposal he gets and rejecting all the others is not consistent with any list of preferences. In fact, different plays of the game induce different orders of proposals; thus, depending on the play of the game, $w$ 's first proposal may be from, say, $f$ and $f^{\prime}$. It follows that $f$ may be revealed preferred to $f^{\prime}$ by $w$ or vice-versa, which clearly cannot be consistent with a preference list.

As for notation, actions are taken at decision nodes, typically denoted by $x$. A strategy profile $\sigma$ specifies a strategy for each agent; we sometimes write $\sigma=\left(\sigma_{v}, \sigma_{-v}\right)$, where $\sigma_{v}$ denotes the strategy of $v$ and $\sigma_{-v}$ denotes the strategy profile of the other agents. Preference strategies will be denoted by the corresponding preference profile- $Q_{v}$, for example, is a preference strategy for $v$-while $P_{v}$ always denotes $v$ 's true preferences. A sequence of firms $o$ and a strategy profile $\sigma$ determine a play of the game, denoted by $\pi$.

\subsection{Random Matching and Ordinal Nash Equilibria}

In the Decentralized Game, different plays of the game with the same strategy profile may yield different output matchings, depending on the order of proposals. This applies even in the case that agents use preference strategies, as the following example illustrates.

Example 1 The outcome depends on the selection of the order by which firms propose.

\footnotetext{
${ }^{9}$ We refer to Pais (2004) for the analysis of a job matching game where the strategy space is confined to the set of preference strategies.
} 
Let $(F, W, P)$ be a marriage market with $P$ such that

$$
\begin{array}{cc}
P_{w_{1}}=f_{2}, f_{1} & P_{f_{1}}=w_{1}, w_{2} \\
P_{w_{2}}=f_{1}, f_{3}, f_{2} & P_{f_{2}}=w_{2}, w_{1} \\
& P_{f_{3}}=w_{2} .
\end{array}
$$

Note that the unique stable matching for this market is $\mu=\left\{\left(f_{1}, w_{2}\right),\left(f_{2}, w_{1}\right)\right\}$. Now consider the Decentralized Game with $\mu^{I}=\left\{\left(f_{1}, w_{2}\right)\right\}$ when agents play according to their true preferences $P$.

Start by considering the case in which $f_{3}$ is the first to make an offer. Given that $f_{3}$ is using $P_{f_{3}}$, it proposes to the only acceptable worker, $w_{2}$, and $w_{2}$ rejects this proposal, as he is initially matched to $f_{1}$, the best firm on his list. Then, it may be the case that either $f_{1}$ 's or $f_{2}$ 's opportunity comes. Let us say $f_{1}$ makes an offer; it proposes to $w_{1}$, the first worker in $P_{f_{1}}$, who is currently unmatched and thus accepts the proposal. Once this proposal is accepted, $w_{2}$ is left unmatched. Hence, when $f_{2}$ is given the chance to propose, $w_{2}$ accepts its offer. In the following moves every firm passes its turn, so that the game ends with the final non-stable matching $\hat{\mu}=\left\{\left(f_{1}, w_{1}\right),\left(f_{2}, w_{2}\right)\right\}$.

Nevertheless, if the first randomly chosen firm is $f_{2}$, its proposal to $w_{2}$ is refused, as this worker is still matched to $f_{1}$ and $f_{1}$ is preferred to $f_{2}$ in $P_{w_{2}}$. The next firm to propose can either be $f_{1}, f_{2}$, or $f_{3}$. Assume $f_{2}$ is the first to propose. It proposes to $w_{1}$, the second worker on its list, and $w_{1}$ accepts. Next, if $f_{1}$ 's turn comes, it proposes to $w_{1}$, who rejects this offer, since he is matched to his top choice $f_{2}$. So imagine $f_{1}$ is called to propose once more, tendering an offer to $w_{2}$, who accepts it. When finally $f_{3}$ proposes to $w_{2}$, he rejects the offer, given that he is already holding the highest ranked firm in his preference list. This play of the game terminates when the three firms are given the chance to pass their turns and the matching 
$\mu=\left\{\left(f_{1}, w_{2}\right),\left(f_{2}, w_{1}\right)\right\}$ is reached as the outcome of the game.

Given an initial matching and a strategy profile, all the uncertainty on the order of play as described above is fully translated into a probability distribution over the set of matchings. Hence, fix a probability distribution on $O$ and take an initial matching $\mu^{I}$, a preference profile $P$, and an arbitrary worker $w$ (what follows also holds for a representative firm, with obvious modifications). We will let $\widetilde{D G}{ }^{\mu^{I}}[\sigma]$ denote the probability distribution over the set of matchings induced by the Decentralized Game starting from $\mu^{I}$ when the strategy profile $\sigma$ is used and $\widetilde{D G}^{\mu^{I}}[\sigma](w)$ is the distribution that $\widetilde{D G}^{\mu^{I}}[\sigma]$ induces over $F \cup\{w\}$. The expression $\operatorname{Pr}\left\{\widetilde{D G}^{\mu^{I}}[\sigma]=\mu\right\}$ represents the probability that $\mu$ is the final matching of the Decentralized Game with the strategy profile $\sigma$. Moreover, $\operatorname{Pr}\left\{\widetilde{D G}{ }^{\mu^{I}}[\sigma](w) R_{w} v\right\}$ is the probability that, in the Decentralized Game, $w$ obtains a partner at least as good as $v$ when $\sigma$ is adopted. Observe that these probabilities rest on the probability distribution on $O$, but all the results that follow hold regardless of this lottery.

To address strategic questions we need to develop ideas about what constitutes a "best decision" to be taken by an agent. With this purpose in mind, let $\sigma$ be a strategy profile and again consider $w \in W$. We say that, given $\sigma_{-w}$, the strategy $\sigma_{w}$ stochastically $P_{w^{-}}$ dominates $\sigma_{w}^{\prime}$ in the Decentralized Game if, for all $v \in F \cup\{w\}, \operatorname{Pr}\left\{\widetilde{D G}^{\mu^{I}}\left[\sigma_{w}, \sigma_{-w}\right](w) R_{w} v\right\} \geq$ $\operatorname{Pr}\left\{\widetilde{D G}^{\mu^{I}}\left[\sigma_{w}^{\prime}, \sigma_{-w}\right](w) R_{w} v\right\}$. Thus, for any level of satisfaction, the probability that $w$ 's match exceeds that level of satisfaction is greater under $\widetilde{D G}^{\mu^{I}}\left[\sigma_{w}, \sigma_{-w}\right]$ than under $\widetilde{D G}^{\mu^{I}}\left[\sigma_{w}^{\prime}, \sigma_{-w}\right]$. This provides the basis for the solution concepts we will adopt throughout the paper.

Definition 1 Let $(F, W, P)$ be a matching market and let $\mu^{I}$ be the initial matching. The profile of strategies $\sigma$ is an ordinal Nash equilibrium (ON equilibrium) in the Decentralized Game if, for each player $v$ in $V, \sigma_{v}$ stochastically $P_{v}$-dominates every alternative strategy $\sigma_{v}^{\prime}$ 
given $\sigma_{-v}$.

Thus, by using a strategy other than $\sigma_{v}, v$ will not be able to strictly increase the probability of obtaining any $v^{\prime}$ (an agent with whom it may end up matched) and all agents ranked higher than $v^{\prime}$ in its true preference list, $P_{v}$. This means that we will be concerned in finding a profile of strategies $\sigma$ with the property that, once adopted by the agents, no one can profit by unilaterally deviating for all possible utility representations of the agents' preferences.

Finally, the notion of ordinal Nash equilibrium can be refined to account for the dynamic nature of the Decentralized Game.

Definition 2 Let $(F, W, P)$ be a matching market and let $\mu^{I}$ be the initial matching. The profile of strategies $\sigma$ is an ordinal subgame perfect Nash equilibrium (OSPN equilibrium) in the Decentralized Game if it induces an ordinal Nash equilibrium in every subgame of the Decentralized Game.

\section{Equilibrium analysis}

We begin this section by exploring the relationship between ordinal Nash and subgame perfect ordinal Nash equilibria.

Proposition 1 Let $|F| \geq 2$. Then, no information set is a singleton.

Lemma 1 Let $|F| \geq 2$. Let $x$ and $x^{\prime}$ be the two last decision nodes of the play of the game $\pi$, such that $x^{\prime}$ precedes $x$. Then, $x$ and $x^{\prime}$ belong to two different firms and both firms choose the action "pass" at these nodes. 
Proof. First, notice that the game ends when every firm has sequentially chosen "pass." Given that $x$ and $x^{\prime}$ precede the terminal node reached with $\pi$ and that $|F| \geq 2$, it follows that the action taken at these nodes must be "pass." Now suppose, by contradiction, that both $x$ and $x^{\prime}$ are firm $f$ 's decision nodes. Since, when $\pi$ is considered, the game ends after $f$ chooses "pass" at $x$, every firm other than $f$ must have chosen "pass" in the nodes that precede $x$. Hence, every firm other than $f$ has passed its turn in the nodes that precede $x^{\prime}$. The rules of the game thus imply that the game ends immediately after $f$ chooses "pass" at $x^{\prime}$ and we reach a contradiction: $x$ is not a decision node.

Lemma 2 Let $|F| \geq 2$. Let $\pi$ be a play of the game and let $x$ be a node of $f$ reached along $\pi$, such that the game does not end after $f$ 's choice at $x$ along $\pi$. Then, there exists a firm $f^{\prime}$ that still has a chance to act in $\pi$.

Proof. Immediate from Lemma 1.

Proof of Proposition 1. Let $x$ be a node that belongs to $f$ in $\pi$ when nature draws the sequence $o$. Let $f$ 's move at $x$ correspond to the $k$ th element of $o$. We will prove that there exists a sequence $o^{\prime}$ and a node $x^{\prime}$ reached when nature draws $o^{\prime}$, such that $x$ and $x^{\prime}$ belong to the same information set.

First, assume that the game does not end after $f$ 's choice at $x$ along $\pi$. By Lemma 2, there exists a firm $f^{\prime}$ that still has the chance to act along $\pi$. Now let $o^{\prime}$ be a sequence whose $k$ first elements are the same as those in $o$, but that differs from $o$ in that $f$ is inserted in position $k+1$ and all the remaining elements are identical. Consider any play of the game where nature draws $o^{\prime}$ and every agent chooses exactly the same actions as along $\pi$ up to the point where $o_{k+1}^{\prime}$ is called to play. Let $x^{\prime}$ be the node corresponding to $f^{\prime}$ 's move in position 
$k$ of the sequence $o^{\prime}$. It is clear that $x^{\prime}$ belongs to the same information set as $x$, since every action, except for the unobservable nature's move, is the same along $\pi$ and $\pi^{\prime}$.

Now let $x$ be a node of $f$, reached along $\pi$, such that $f$ 's action at $x$ is the last action in $\pi$. By Lemma 1, there exists a firm $f^{\prime} \neq f$ that has had the chance to move immediately before $f$ moves at $x$, i.e., in position $o_{k-1}$ of the sequence, and both have chosen "pass." Now let $o^{\prime}$ be a sequence whose first $k-1$ elements coincide with those of $o$, but where $f^{\prime}$ occupies the position $o_{k}$ and $f$ occupies the position $o_{k+1}$. Consider the play of the game $\pi^{\prime}$ where nature draws $o^{\prime}$, every agent up to the element $k-1$ in the sequence chooses exactly the same action as in $\pi$, and $f^{\prime}$ chooses "pass" when called to play at the $k$ th position of the sequence. Let $x^{\prime}$ be the node reached in $\pi^{\prime}$ where $f$ acts in position $k+1$. Since $f$ cannot observe nature's moves nor $f^{\prime \prime}$ s action, it holds exactly the same information in both $x$ and $x^{\prime}$. Hence, $x^{\prime}$ belongs to the same information set as $x$.

Now consider $\pi$ where nature draws $o$ and along which some worker $w$ may accept or reject a proposal made by firm $f$. Let $x$ be the node where $w$ acts and let $f$ 's proposal correspond to the $k$ th element of $o$. Lemma 1 ensures that the game does not end after $w$ 's move at $x$. Hence, let $o^{\prime}$ be any sequence whose $k$ first elements are the same as those in $o$, but such that the elements in position $k+1$ are different. Define $\pi^{\prime}$ as a play of the game in which nature draws $o^{\prime}$ and every other player chooses the same actions as along $\pi$ up to the point where $w$ reacts to $f$ 's proposal. Let $x^{\prime}$ be the node where $w$ takes such decision. Since nature's draws are not observable, w's information is exactly the same in $x$ and in $x^{\prime}$. It follows that the information set containing $x$ is not a singleton.

An immediate implication of this result is that the set of ordinal Nash and subgame perfect ordinal Nash equilibria coincide. In fact, given that all information sets are non-singletons, the 
Decentralized Game has no proper subgames. It may be conjectured that this is due to the low information environment we have assumed. And there are labor markets in which agents may become aware of events that do not affect them directly - acquaintances and social networks in general may play an important role. However, considering an enriched information environment where agents perceive all the offers that are made, as well as the proposed workers' reactions, the arguments in the above proof remain valid, as long as nature's move remains unobservable. Roughly speaking, for every decision node $x$ along some play of the game that includes a draw of nature $o$, it is always possible to find a decision node $x^{\prime}$ belonging to the same information set of $x$ by building a different play of the game in the following way: add a single firm to $o$, let it choose "pass" in its new decision node, and let agents choose exactly the same actions as in the original play in every other node. The conclusion follows since every proposal, acceptance, and rejection is made respecting the original order. Hence, even in this extreme case, ordinal subgame perfect Nash coincide with ordinal Nash equilibria. In what follows, we will refer to these concepts indistinctly as ordinal equilibria.

The following theorem is the main result of this section. Individual rationality is an obvious necessary condition that every ordinal equilibrium outcome must fulfill. Here, we state that under every ordinal equilibrium play of the Decentralized Game where firms use lists of preferences, some form of stability is preserved. To be more precise, every matching that can be obtained under such a play is stable for the same profile of preferences. The following remark is used in the proof of the theorem.

Remark 1 When using a preference strategy, a firm will not fire a worker it proposed to nor exchange him for another worker along any play of the Decentralized Game. In fact, when a proposal is made, the firm reveals that this particular worker is the best among all who have 
not rejected it. If the worker accepts, the only occasion under which the firm makes a proposal again is when the worker it holds resigns from his position.

Theorem 1 Let $\mu^{I}$ be an individually rational input matching for $\left(F, W,\left(Q_{F}, P_{W}\right)\right)$. Assume that the strategy profile $\sigma=\left(Q_{F}, \sigma_{W}\right)$ is an ordinal equilibrium in the Decentralized Game. Then, the probability distribution obtained over the set of matchings is such that every element in its support is a member of $S\left(Q_{F}, P_{W}\right)$.

Proof. Suppose that $\left\{\mu_{1}, \ldots, \mu_{k}\right\}$ is the support of the distribution induced over the set of matchings when agents use $\sigma$. Assume that for some $i \in\{1, \ldots, k\}, \mu_{i} \notin S\left(Q_{F}, \sigma_{W}\right)$. We will prove that $\sigma$ is not an ordinal equilibrium.

Let $\pi$ be a play of the game that results in $\mu_{i}$. To start, notice that for every firm $f$ it must be the case that its assignment, $\mu_{i}(f)$, is acceptable with respect to $Q_{f}$. In fact, once using $Q_{f}, f$ never proposes, under any play of the game, to a worker that, according to $Q_{f}$, is considered worse than being unmatched. On the other hand, every worker must consider his partner acceptable with respect to $P$. Assume that this is not the case and that there exists a worker, say $w$, such that $w P_{w} \mu_{i}(w)$. Individual rationality of the matching $\mu^{I}$ implies $\mu_{i}(w) \neq \mu^{I}(w)$. Hence, $\sigma_{w}$ must include, at some point along $\pi$, accepting $\mu_{i}(w)$ 's proposal. Now take an alternative strategy $\hat{\sigma}_{w}$ according to which no offer is accepted by $w$. By using $\hat{\sigma}_{w}, w$ may end up unmatched or, if initially matched, keep his original partner $\mu^{I}(w)$, but he is never assigned to a firm considered unacceptable under $P_{w}$. Thus, the following holds:

$$
1=\operatorname{Pr}\left\{\widetilde{D G}^{\mu^{I}}\left[\hat{\sigma}_{w}, \sigma_{-w}\right](w) R_{w} w\right\}>\operatorname{Pr}\left\{\widetilde{D G}^{\mu^{I}}[\sigma](w) R_{w} w\right\}
$$

and $\sigma_{w}$ is not a best reply to $\sigma_{-w}$. 
We have proved that $\mu_{i}$ is individually rational. Thus, there must exist a blocking pair for $\mu_{i}$ when the preference profile $\left(Q_{F}, P_{W}\right)$ is considered. Let us say $(f, w)$ blocks $\mu_{i}$, i.e., $f P_{w} \mu_{i}(w)$ and $w Q_{f} \mu_{i}(f)$. This implies that $f$ proposed to $w$ and, by Remark 1 , was rejected by $w$ in the course of $\pi$. Hence, $\sigma_{w}$ includes rejecting $f$ in at least one of $w$ 's information sets. Now, define $\hat{\sigma}_{w}$ as the strategy according to which $w$ chooses the same actions as under $\sigma_{w}$ at every information set, except for those that lead to rejecting $f$. When using $\hat{\sigma}_{w}$, if $f$ proposes to $w, w$ accepts this proposal and rejects every subsequent offer.

First, let us prove that the probability of being assigned to $f$ is strictly higher under $\hat{\sigma}_{w}$ than under $\sigma_{w}$. Recall that $\pi$ is a play of the game leading to $\mu_{i}$ and let $o$ be nature's move in $\pi$. We know that firm $f$ must have proposed to $w$ along $\pi$. If, instead of using $\sigma_{w}, w$ deviates and acts according to $\hat{\sigma}_{w}$, by Remark 1, w will end up matched to $f$ when nature draws $o$ and $\sigma_{-w}$ is used. Now let $\pi^{\prime}$ be the play of the game in which nature draws $o^{\prime}$, with $o^{\prime} \neq o$, and players use $\left(\hat{\sigma}_{w}, \sigma_{-w}\right)$. If $f$ does not propose to $w$ along $\pi^{\prime}, w$ acts exactly as if using $\sigma_{w}$ and ends up matched to the same partner as when nature draws $o$ and players use $\sigma$. Otherwise, $f$ and $w$ are matched in the final matching. It follows that the probability of having $f$ and $w$ matched is strictly increased when $w$ uses $\hat{\sigma}_{w}$.

In order to prove $\sigma_{w}$ is not a best reply to $\sigma_{-w}$, assume, without loss of generality, that $P_{w}=f_{1}, f_{2}, \ldots, f_{m-1}, f, f_{m+1}, \ldots, w, \ldots, f_{n}$. Consider a firm $f_{j}$, with $j=1, \ldots, m-1$, and consider all the plays of the game where $\sigma$ is used and where $w$ and $f_{j}$ end up together in the final matching. Some of these plays may not give $f_{j}$ assigned to $w$ when he deviates and acts according to $\hat{\sigma}_{w}$. However, the only occasion under which this happens is when $w$ obtains a proposal from $f$ and ends up matched to $f$. Hence, the probability of having $w$ matched to $f$ 
or to a firm he considers better than $f$ is strictly increased when $w$ uses $\hat{\sigma}_{w}$. We have

$$
\operatorname{Pr}\left\{\widetilde{D G}^{\mu^{I}}\left[\hat{\sigma}_{w}, \sigma_{-w}\right](w) R_{w} f\right\}>\operatorname{Pr}\left\{\widetilde{D G}^{\mu^{I}}[\sigma](w) R_{w} f\right\}
$$

contradicting that $\sigma$ is an ordinal equilibrium.

The importance of this result lies in two of its implications. Since the set of unmatched agents is the same for every matching that is stable in a matching market (McVitie and Wilson, 1970, and Roth, 1982), the same agents remain unmatched in every possible outcome of an ordinal equilibrium where firms use lists of workers to guide their decisions. Moreover, when we focus on equilibria where firms act according to their true preferences, stability with respect to the true preferences is guaranteed. Such straightforward form of behavior can be justified. In some settings, firms obey objective criteria when selecting whom to hire (e.g., universities select students according to their grades, some firms choose their workers basing on scores given by a recruiting agency, student placement mechanisms assign students to public schools according to the area of residence,...). Even when firms are not constrained to follow such rules, hiring new workers embodies a process of aggregating the opinions of different individuals that compose a recruiting committee; hence, it may be that a list of workers is fixed and all decisions are myopically taken basing on that list. Having to decide what to do next at each moment in time may be a less plausible form of behavior. Finally, reverting to the true preferences is always an easy resort, given the multiplicity of available strategies and the complexity of the environment.

Ordinal equilibria always exist when the initial matching is individually rational. In particular, the following results show the existence of ordinal equilibria where firms use preference strategies. 
Definition 3 Let $\mu^{I}$ be an arbitrary matching. We say that $\mu$ is individually rational with respect to $\mu^{I}$ if $\mu \in I R(P)$ and if, for all $f \in F, w^{\prime}=\mu^{I}(f) P(f) \mu(f)$, implies $\mu\left(w^{\prime}\right) \neq w^{\prime}$.

We will denote by $I R^{\mu^{I}}(P)$ the set of all individually rational matchings with respect to $\mu^{I}$. For illustration, in the particular case that $\mu^{I}$ is the empty matching, the set of all individually rational matchings with respect to this initial matching coincides with the set of individually rational matchings (i.e., $\left.I R^{\varnothing}(P)=I R(P)\right) .{ }^{10}$

Proposition 2 Let $\mu^{I}$ be an individually rational matching for $(F, W, P)$. Then, $S(P)$ is a subset of $I R^{\mu^{I}}(P)$.

Proof. Consider $\mu \in S(P)$. We will prove that $\mu \in I R^{\mu^{I}}(P)$ using a contradiction argument. Assume that $\mu \in S(P)$. By definition of stability, this implies $\mu \in I R(P)$, but assume that there exists a firm $f$ such that $w^{\prime}=\mu^{I}(f) P_{f} \mu(f)$ and $\mu\left(w^{\prime}\right)=w^{\prime}$. Stability of $\mu$ implies that $w^{\prime} P_{w^{\prime}} f$ and we get a contradiction: $\mu^{I}$ is not individually rational. Therefore, every stable matching is an element of $I R^{\mu^{I}}(P)$.

Since a stable matching exists for every marriage market (Gale and Shapley, 1962), $I R^{\mu^{I}}(P)$ is not empty for every individually rational matching $\mu^{I}$.

Proposition 3 Let $\mu^{I}$ be an individually rational matching for $(F, W, P)$ and let $\mu \in I R^{\mu^{I}}(P)$. Then, there exists an ordinal equilibrium $\sigma=\left(Q_{F}, \sigma_{W}\right)$ in the Decentralized Game that leads to $\mu$ with probability one.

Proof. Define $Q_{f}=\mu(f)$, for every firm $f$ and let $\sigma_{w}=Q_{w}=\mu(w)$. It is clear that every play of the game with the profile $\sigma$ will lead to the output matching $\mu$.

\footnotetext{
${ }^{10}$ This holds since if $f P(f) \mu(f)$, then $\mu(f) \neq f$.
} 
Let us show that for every firm $f, Q_{f}$ is a best reply to $Q_{-f}$. First, as long as $\mu(f) \neq \mu^{I}(f)$, $f$ never holds its initial match under $\mu$. Indeed, it is clear that if $\mu^{I}(f) P_{f} \mu(f)$, then $\mu^{I}(f)$ receives and accepts another firm's proposal (and in the case that $\mu(f) P_{f} \mu^{I}(f), \mu^{I}(f)$ is not a temptation). Hence, when $\mu(f) \in W$, given that the only worker willing to accept $f$ 's proposal is $\mu(f)$, the only choice $f$ can actually make is between being assigned to this worker or staying alone. From individual rationality we have $\mu(f) P_{f} f$ which implies that $f$ will not be able to profit from deviating from $Q_{f}$. Obviously, for $f$ such that $\mu(f)=f$, no worker accepts $f$ 's proposal and it can do no better than staying alone.

Finally, for any $w, \sigma_{w}$ is a best reply to $\sigma_{-w}$. In fact, given firms' strategies, $w$ gets at most one proposal and, considering $\mu$ is individually rational, the best he can do is to accept it. This completes the proof.

One particular case worth exploring is the case in which the starting point is the empty matching. The Decentralized Game then becomes a stylized model of an entry-level labor market without commitment, where cohorts of vacant positions and cohorts of candidates become simultaneously available, and decisions are taken in a decentralized way. It turns out that starting from the empty matching allows us to take the analysis farther.

Proposition 4 Let $\mu^{I}$ be the empty matching and let $\mu \in S(P)$. Then, there exists an ordinal equilibrium in the Decentralized Game where firms reveal their true preferences that yields $\mu$ with probability one.

Proof. Let $\sigma=\left(P_{F}, \sigma_{W}\right)$ and define $\sigma_{w}$ as follows. For every worker $w$ matched under $\mu$, $\sigma_{w}$ is the strategy of accepting only $\mu(w)$ and rejecting every other proposal, while it leads to the rejection of all proposals, without exception, when $w$ is unmatched under $\mu$. 
We start by showing that the profile of strategies $\sigma$ always leads to the matching $\mu$, i.e., $\operatorname{Pr}\left\{\widetilde{D G}^{\mu^{I}}[\sigma]=\mu\right\}=1$. If this is not the case, then there exists a play of the game leading to $\widehat{\mu} \neq \mu$. But this is equivalent to having a firm, say $f$, that ends up matched to a partner, $\widehat{\mu}(f)$, different from $\mu(f)$ for some instance of the game. Given that $f$ is unmatched in the initial matching and that the only worker willing to accept $f$ is $\mu(f)$, we must have $\widehat{\mu}(f)=f$ (as long as $f \neq \mu(f)$; otherwise it must be the case that $\widehat{\mu}(f)=\mu(f)$ and we have a contradiction). So assume that $\widehat{\mu}(f)=f$. Since $\mu(f)$ would accept $f$ 's proposal and $f$ is acting according to its true preferences, it must be the case that $f P_{f} \mu(f)$. Hence, $\mu(f)$ is not acceptable and the stability of $\mu$ is contradicted.

Let us now prove that, for every firm $f, P_{f}$ stochastically $P_{f}$-dominates every other strategy $\sigma_{f}$. We will start by considering the case in which $\mu(f) \neq f$. Given that the only worker who is willing to accept $f$ is $\mu(f)$, by choosing its strategy appropriately, $f$ can either be alone or hold $\mu(f)$ under the output matching. By stability of $\mu, \mu(f) P_{f} f$; since truth telling guarantees that $\mu(f)$ is assigned to $f$ with probability one, $f$ cannot improve by switching its strategy. In the case that $\mu(f)=f$, no worker accepts its proposal, and the best it can achieve is staying unmatched. It follows that $f$ cannot do better than being assigned to $\mu(f)$ and $P_{f}$ stochastically $P_{f}$-dominates every other strategy $\sigma_{f}$.

Now take the case of an arbitrary worker, $w$. Suppose, by contradiction, that $\sigma_{w}$ does not stochastically $P_{w^{-d o m i n a t e ~ s t r a t e g y ~}} \hat{\sigma}_{w}$. This implies that $\operatorname{Pr}\left\{\widetilde{D G}^{\mu^{I}}\left[P_{F}, \hat{\sigma}_{w}, \sigma_{-w}\right](w) R_{w} \mu(w)\right\}=$ 1 and that there exists a firm, say $f$, such that the following holds: $\operatorname{Pr}\left\{\widetilde{D G} \mu^{I}\left[P_{F}, \hat{\sigma}_{w}, \sigma_{-w}\right](w)=\right.$ $f\}>0$ and $f P_{w} \mu(w)$. But this means that, for some draw of nature, $f$ approaches $w$ before making an offer to $\mu(f)$. In fact, it cannot be the case that $f$ proposes to $\mu(f)$ first and he does not accept it, as $\mu(f)$ is acting according to his original strategy, $\sigma_{\mu(f)}$, defined above. Thus, $f$ 
must prefer $w$ to $\mu(f)$. However, in this case $(f, w)$ forms a blocking pair for $\mu$, contradicting the fact that $\mu$ is stable.

Hence, in a decentralized entry-level labor market every stable matching can be reached as the outcome of an ordinal equilibrium play of the game where firms stick to their true rankings. The (partially) converse statement is given by Theorem 1, ensuring that every such ordinal equilibrium guarantees stability. These results may be viewed as an extension of some known features of the game induced by Gale and Shapley's centralized mechanism (Roth, 1984b), where the underlying strategy space is confined to the set of preference strategies.

\section{Discussion}

In this section we put our results in perspective and discuss some of the underlying assumptions.

As mentioned in the Introduction, centralized procedures have been introduced in many matching markets in response to certain market failures. It has been argued that the stability of the mechanisms employed is crucial for their success. In fact, those centralized procedures that achieved stable outcomes resolved the market failures, while those producing unstable outcomes continued to fail. ${ }^{11}$ Since many matching markets do not employ centralized matching procedures, and yet are not observed to experience such problems, we can suspect that some markets may reach stable outcomes by means of decentralized decision making without commitment. Theorem 1 provides support to this conjecture. To make things clear, let us return to Example 1. We have seen that some plays of the game lead to unstable outcomes for the true preferences (the matching $\hat{\mu}$ is not stable). Nevertheless, Theorem 1 implies that

\footnotetext{
${ }^{11}$ See, for example, Roth (1984a, 1990, 1991).
} 
if we expect agents to use equilibrium strategies and, by best-replying, firms faithfully reveal their true preferences, then a stable matching is reached. Hence, if equilibrium predictions are to be taken seriously, the success of some decentralized markets is explained.

It is now probably worth discussing the robustness of the results to some changes in the rules of the game. First, as already mentioned, the simplifying assumption that firms do not repeat offers ensures that the game is finite. Still, admitting that firms are persistent does not compromise the above results, as long as we allow for a finite number of repetitions.

Second, throughout the game matchings are formed and dissolved as agents act in what they perceive to be their own best interest. We may think of this as a mere negotiation process, where no contracts are signed and where these temporary matchings would be the ones prevailing should negotiations suddenly stop. Alternatively, considering that provisional matchings are indeed consummated amounts to assuming that agents are free to recontract without any restrictions whatsoever. In the other extreme, we can consider that it is too costly to fire a worker. Hence, only firms with vacancies will actually make proposals and the Decentralized Game falls in the realms of Blum, Roth, and Rothblum's analysis. Blum, Roth, and Rothblum (1997) study how markets for senior positions may be re-stabilized after new firms have been created or workers have retired. In fact, stability for the true preferences is achieved in every equilibrium where firms act according to their true preferences, as long as the starting point is a firm-quasi-stable matching, i.e., a matching whose stability has been disrupted by the creation of a new position or the retirement of a worker. Hence, Theorem 1, which allows for having any individually rational as an initial matching, no longer holds. The validity of Proposition 3 is also compromised: if we start form an initial matching where every firm is matched, no firm will be allowed to hire a new worker and the initial matching 
situation will be preserved, independently of the strategies used. In this setting, the majority of the results on equilibria depend on having a firm-quasi-stable matching as a starting point.

A different issue concerns providing workers with the initiative to quit and to propose. In some real labor markets, not only firms, but also workers may defy their preferred firms and we may account for this in the Decentralized Game. Hence, suppose that at each moment in time, an agent, either a firm or a worker, is randomly selected and makes an offer to someone in the other side of the market to whom it has never proposed to nor received a proposal from. The agent that receives the offer can only accept, or reject and keep his former partner, if a former partner existed. The game ends when every agent in the market passes its turn. It turns out that, starting from an arbitrary matching, every individually rational matching can be obtained in an ordinal equilibrium play of the game, so that the scope of Proposition 3 is enhanced. In what Theorem 1 and its implications are concerned, stability is robust to sophisticated behavior by one side of the market, provided that the other side acts in accordance with the true preferences. To be precise, every matching sustained at an ordinal equilibrium is stable with respect to the true preferences whenever firms (respectively workers) faithfully transmit their preferences and workers (respectively firms) behave strategically by using strategies that may reveal different orderings of the other side of the market in different executions of the algorithm. Finally, in the particular case that the initial matching is the empty matching, every stable matching can be reached with probability one in an equilibrium where one side of the market truthfully reveals its preferences. ${ }^{12}$

\footnotetext{
${ }^{12} \mathrm{~A}$ formal statement of these results and their proofs are given in the Appendix, in Propositions 5, 6, and 7.
} 


\section{Concluding Remarks}

The present paper attempts to extend the two-sided matching theory by constructing a game that mimics the behavior of some decentralized labor markets. Equilibrium analysis in a random context is performed at the expense of using an ordinal equilibrium concept that allows for obtaining some interesting results. Namely, equilibria where firms use preference strategies always exist and lead to matchings that preserve stability for a particular profile of preferences. Furthermore, when we consider an ordinal equilibrium where firms act truthfully, stability for the true preferences is achieved in every outcome matching. This fact may account for the success of some decentralized labor markets. A case of particular interest has the empty matching as the starting point of the game. Here we give a fairly complete characterization of ordinal equilibria.

It is natural to ask to what extent the stylized model constructed here can serve as a description of a real decentralized labor market. The marriage model is perhaps too simple. Aside from the assumption that each firm has a unique position to fill, the important unrealistic feature lies in considering that the salary associated with each position is a fixed part of the job description, rather than something to be negotiated between each firm and prospective worker. Furthermore, the concept of ordinal equilibrium - justified by the ordinal nature of agents' preferences - is quite demanding. It thus remains important to explore models where these assumptions are relaxed and a milder solution concept is used, even though we believe that the present analysis provides a good starting point to understand the functioning of some decentralized labor markets. 


\section{Appendix}

In this section we extend some of the above results to the case in which both sides of the market are able to tender offers. First, it can easily be shown that no information set is a singleton, as long as there are at least two agents in the market, i.e., $|V| \geq 2 .{ }^{13}$ It follows that subgame perfect ordinal Nash equilibria coincide with ordinal Nash equilibria. The remaining results are proved in what follows.

Proposition 5 Let $\mu^{I}$ be an arbitrary matching in $(F, W, P)$ and let $\mu \in I R(P)$. Then, there exists an ordinal equilibrium in the Decentralized Game that leads to $\mu$ with probability one.

Proof. Let $Q_{v}=\mu(v)$, for all $v \in V$. Clearly, every play of the game with strategy profile $Q$ leads to $\mu$. We will show that $Q$ is an ordinal equilibrium. In the case that $v$ is such that $\mu(v) \neq v$, the only agent that proposes to or accepts a proposal from $v$ is $\mu(v)$. Hence, no deviation will improve $v$ 's match. Otherwise, for $v$ such that $\mu(v)=v$, no agent is willing to match $v$. As before, by switching strategy, $v$ cannot end up matched and improve his position.

In what follows, we extend Theorem 1 . The result is stated for equilibria in which firms use preference strategies and workers are allowed to have other forms of behavior. Note however that we restrict to equilibria where a worker's strategy is consistent with a list of preferences along each play of the game (even though it may correspond to incompatible lists when different plays of the game are considered). A similar result, where the roles of firms and workers are

\footnotetext{
${ }^{13}$ The reasoning behind the proof of Proposition 1 remains valid, but instead of analysing decision nodes that belong to firms and to workers as separate cases, the distinction to be made is between nodes where proposals are issued, and those where acceptances or rejections take place.
} 
interchanged, can be proved.

Proposition 6 Let $\mu^{I}$ be an arbitrary matching in $(F, W, P)$. Assume that the strategy profile $\sigma=\left(Q_{F}, \sigma_{W}\right)$ is an ordinal equilibrium in the Decentralized Game, where $\sigma_{w}$ is consistent with a list of preferences in each play of the game, for all $w \in W$. Then, the probability distribution obtained over the set of matchings is such that every element in its support is a member of $S\left(Q_{F}, P_{W}\right)$

Proof. Suppose that $\left\{\mu_{1}, \ldots, \mu_{k}\right\}$ is the support of the distribution induced over the set of matchings when agents use $\sigma$ and assume that for some $i \in\{1, \ldots, k\}, \mu_{i} \notin S\left(Q_{F}, \sigma_{W}\right)$. We will prove that $\sigma$ is not an ordinal equilibrium.

We will denote by $\pi$ a play of the game leading to $\mu_{i}$. To start, notice that for every firm $f$ it must be the case that its assignment, $\mu_{i}(f)$, is acceptable with respect to $Q_{f}$. In fact, once using $Q_{f}, f$ never proposes to nor accepts a proposal from a worker that, according to $Q_{f}$, is considered worse than being unmatched. On the other hand, every worker must find his partner acceptable. Assume that this is not the case and that there exists a worker, say $w$, such that $w P_{w} \mu_{i}(w)$. Now take an alternative strategy $\hat{\sigma}_{w}$ according to which $w$ resigns from $\mu^{I}(w)$ - if $w$ is initially matched - and accepts no offers. By using $\hat{\sigma}_{w}, w$ ends up unmatched in every play of the game. Hence, $1=\operatorname{Pr}\left\{\widetilde{D G}^{\mu^{I}}\left[\hat{\sigma}_{w}, \sigma_{-w}\right](w) R_{w} w\right\}>\operatorname{Pr}\left\{\widetilde{D G}^{\mu^{I}}[\sigma](w) R_{w} w\right\}$ and $\sigma_{w}$ is not a best reply to $\sigma_{-w}$.

Individual rationality of $\mu_{i}$ of $\left(Q_{F}, P_{W}\right)$ is proven. Thus, there must exist a blocking pair for $\mu_{i}$ when the preference profile $\left(Q_{F}, P_{W}\right)$ is considered. Let us say $(f, w)$ blocks $\mu_{i}$, i.e., $f P_{w} \mu_{i}(w)$ and $w Q_{f} \mu_{i}(f)$. This implies that, in the course of $\pi$, either (i) $f$ proposed to $w$ or (ii) $w$ proposed to $f$. If (i) holds, by Remark $1, f$ was rejected by $w$ and we can prove that $\sigma_{w}$ 
is not a best-reply to $\sigma_{-w}$ using the same arguments as in the proof of Theorem 1 . Otherwise, in case (ii), since $w$ uses a strategy that is consistent with a list of preferences under $\pi, f$ must have rejected $w$. (The reasoning behind this relies in arguments similar to those of Remark 1.) In this case we can find a successful deviation for $f$. In fact, define $\hat{\sigma}_{f}$ as the strategy according to which $f$ chooses the same actions as under $\sigma_{f}$ at every information set, except for those that lead to rejecting $w$ when $w$ proposes. Hence, when using $\hat{\sigma}_{f}$, if $w$ proposes to $f$ along a play of the game, $f$ accepts this proposal and holds it until the end of this play. For every play of the game in which $w$ does not propose to $f, f$ acts exactly as when using $\sigma_{f}$.

First, we will prove that the probability of being assigned to $w$ is strictly higher under $\hat{\sigma}_{f}$ than under $\sigma_{f}$. Recall that $\pi$ is a play of the game leading to $\mu_{i}$ and let $o$ be nature's move in $\pi$. We know that $w$ must have proposed to $f$ along $\pi$. Once $f$ deviates and acts according to $\hat{\sigma}_{f}, f$ will end up matched to $w$ when nature draws $o$ and $\sigma_{-f}$ is used. Now let $\pi^{\prime}$ be the play of the game in which nature draws $o^{\prime}$, with $o^{\prime} \neq o$, and players use $\left(\hat{\sigma}_{f}, \sigma_{-f}\right)$. If $w$ does not propose to $f$ along $\pi^{\prime}, f$ ends up matched to the same partner as when nature draws $o$ and players use $\sigma$. Otherwise, $f$ and $w$ are matched in the final matching. It follows that the probability of having $f$ and $w$ matched is strictly increased when $f$ uses $\hat{\sigma}_{f}$.

In order to complete the proof that $\sigma_{f}$ is not a best reply to $\sigma_{-f}$, assume, without loss of generality, that $P_{f}=w_{1}, w_{2}, \ldots, w_{l-1}, w, w_{l+1}, \ldots, f$. Consider a worker $w_{j}$, with $j=1, \ldots, l-1$, and consider all the plays of the game where $\sigma$ is used and where $f$ and $w_{j}$ end up together in the final matching. Such plays may not give $w_{j}$ assigned to $f$ when $f$ switches to $\hat{\sigma}_{f}$. However, the only occasion under which this happens is when $f$ obtains a proposal from $w$ and ends up matched to him. Hence, the probability of having $f$ matched to $w$ or to a worker it considers 
better than $w$ is strictly increased when $f$ uses $\hat{\sigma}_{f}$. We have

$$
\operatorname{Pr}\left\{\widetilde{D G}^{\mu^{I}}\left[\hat{\sigma}_{f}, \sigma_{-f}\right](f) R_{f} w\right\}>\operatorname{Pr}\left\{\widetilde{D G}^{\mu^{I}}[\sigma](f) R_{f} w\right\}
$$

contradicting that $\sigma$ is an ordinal equilibrium.

Proposition 7 Let $\mu^{I}$ be the empty matching and let $\mu \in S(P)$. Then, there exists an ordinal equilibrium in the Decentralized Game where one side of the market reveals its true preferences that yields $\mu$ with probability one.

Proof. We analyze the case in which firms act according to their true preferences; the same arguments hold, with the roles of firms and workers reversed, when workers act straightforwardly. Hence, consider $\sigma=\left(P_{F}, \sigma_{W}\right)$ and define $\sigma_{w}$ as follows: if $w$ is matched under $\mu$, $\sigma_{w}$ is the strategy of always choosing "pass" when called to propose and accepting only $\mu(w)$ 's proposal; while if $w$ is such that $\mu(w)=w$, no proposal is made nor accepted by $w$.

We start by showing that the profile of strategies $\sigma$ always leads to the matching $\mu$. If this is not the case, then there exists a play of the game leading to $\widehat{\mu} \neq \mu$. But this is equivalent to having a firm, say $f$, that ends up matched to a partner, $\widehat{\mu}(f)$, different from $\mu(f)$ for some instance of the game. Given that workers make no proposals and that the only one willing to accept $f$ is $\mu(f)$, we must have $\widehat{\mu}(f)=f$ (as long as $f \neq \mu(f)$; otherwise it must be the case that $\widehat{\mu}(f)=\mu(f)$ and we have a contradiction). So assume that $\widehat{\mu}(f)=f$. Since $\mu(f)$ would accept $f$ 's proposal and $f$ is acting according to its true preferences, it must be the case that $f P_{f} \mu(f)$. Hence, $\mu(f)$ is not acceptable and the stability of $\mu$ is contradicted.

Let us now prove that, for every firm $f, P_{f}$ stochastically $P_{f}$-dominates every other strategy $\sigma_{f}$. We will start by considering the case in which $\mu(f) \neq f$. Given that workers do not issue offers and that the only worker who is willing to accept $f$ is $\mu(f)$, by choosing its strategy 
appropriately, $f$ can either be alone or hold $\mu(f)$ under the output matching. By stability of $\mu, \mu(f) P_{f} f$; since truth telling guarantees that $\mu(f)$ is assigned to $f$ with probability one, $f$ cannot improve by deviating. In the case that $\mu(f)=f$, no worker accepts its proposal nor proposes to $f$, and the best it can achieve is staying unmatched. It follows that $f$ cannot do better than being assigned to $\mu(f)$ and $P_{f}$ stochastically $P_{f}$-dominates every other strategy $\sigma_{f}$.

Now take the case of an arbitrary worker, $w$. Suppose, by contradiction, that $\sigma_{w}$ does not stochastically $P_{w}$-dominate a different strategy $\hat{\sigma}_{w}$. Then, $\operatorname{Pr}\left\{\widetilde{D G} \mu^{I}\left[P_{F}, \hat{\sigma}_{w}, \sigma_{-w}\right](w) R_{w} \mu(w)\right\}=$ 1 and that there exists a firm, say $f$, such that the following holds: $\operatorname{Pr}\left\{\widetilde{D G} \mu^{I}\left[P_{F}, \hat{\sigma}_{w}, \sigma_{-w}\right](w)=\right.$ $f\}>0$ and $f P_{w} \mu(w)$. Let $\pi$ be a play of the game where $f$ and $w$ are matched. By stability of $\mu, \mu(f) P_{f} w$, so that $f$ proposes to $\mu(f)$ in the course of $\pi$. Given the outcome matching, $\mu(f)$ rejects $f$ 's proposal. This contradicts the definition of $\mu(f)$ 's strategy.

\section{References}

Blum, Y., A. E. Roth, and U. G. Rothblum (1997): "Vacancy Chains and Equilibration in Senior-Level Labor Markets," Journal of Economic Theory, 76, 362-411.

D’Aspremont, C., And B. Peleg (1988): “Ordinal Bayesian Incentive Compatible Representations of Committees," Social Choice and Welfare, 5, 261-279.

Ehlers, L., AND J. MASsó (2003): "Incomplete Information and Small Cores in Matching Markets," Mimeo, Universitat Autònoma de Barcelona.

Gale, D., and L. S. Shapley (1962): "College Admissions and the Stability of Marriage," American Mathematical Monthly, 69, 9-15. 
Haeringer, G., and M. Wooders (2004): "Decentralized Job Matching," Mimeo, Universitat Autònoma de Barcelona.

Majumdar, D. (2003): "Ordinally Bayesian Incentive Compatible Stable Matchings," Mimeo.

Majumdar, D., And A. Sen (2004): "Ordinally Bayesian Incentive Compatible Voting Rules," Econometrica, 72, 523-540.

McVitie, D. G., And L. B. Wilson (1970): "Stable Marriage Assignment for Unequal Sets," BIT, 10, 295-309.

Niederle, M., and A. E. Roth (2003): "Unraveling Reduces Mobility in a Labor Market: Gastroentrology With and Without a Centralized Match," Journal of Political Economy, forthcoming.

PAIS, J. (2004): "On Random Matching Markets: Properties and Equilibria," Mimeo, Universitat Autònoma de Barcelona.

Roth, A. E. (1982): "The Economics of Matching: Stability and Incentives," Mathematics of Operations Research, 7, 617-628.

_ (1984a): "The Evolution of the Labor Market for Medical Interns and Residents: A Case Study in Game Theory," Journal of Political Economy, 92, 991-1016.

_ (1984b): "Misrepresentation and Stability in the Marriage Problem," Journal of Economic Theory, 34, 383-387.

(1990): “New Physicians: A Natural Experiment in Market Organization," Science, $250,1524-1528$. 
(1991): "A Natural Experiment in the Organization of Entry Level Labor Markets: Regional Markets for New Physicians and Surgeons in the U.K.," American Economic Review, 81, 415-440.

Roth, A. E., And M. A. O. Sotomayor (1990): Two-Sided Matching: A Study in GameTheoretic Modeling and Analysis. Cambridge University Press, Cambridge, England.

Roth, A. E., and J. H. V. Vate (1991): "Incentives in Two-Sided Matching with Random Stable Mechanisms," Economic Theory, 1, 31-44.

Roth, A. E., And X. Xing (1994): "Jumping the Gun: Imperfections and Institutions Related to the Timing of Market Transactions," American Economic Review, 84, 992-1044.

(1997): "Turnaround Time and Bottlenecks in Market Clearing: Decentralized Matching in the Market for Clinical Psychologists," Journal of Political Economy, 105, 284-329. 